\title{
LA SALUD PÚBLICA EN TRANSFORMACIÓN. LA ESTRUCTURACIÓN DE POLÍTICAS SANITARIAS EN CÓRDOBA-ARGENTINA, 1930-1943
}

\author{
María José Ortiz Bergia"
}

Universidad Nacional de Córdoba

\section{RESUMEN}

Esta investigación tiene como objetivo analizar los procesos de estructuración de políticas sanitarias en una provincia argentina a lo largo de los años treinta y comienzos de los cuarenta, poniendo en evidencia la importancia de este período en la generación de cambios en el tratamiento estatal de la salud en el interior del país. Para ello, el trabajo se dedica a estudiar la construcción de las políticas sanitarias y las nuevas capacidades estatales que se desplegaron alrededor del campo de la salud pública en la provincia de Córdoba.

PALABRAS CLAVE: Salud. Políticas Sociales. Intervencionismo. Córdoba. Argentina.

THE TRANSFORMATION PROCESS OF THE PUBLIC HEALTH SYSTEM. THE STRUCTURING OF HEALTH POLICIES IN CÓRDOBA, ARGENTINA, 1930-1943

\begin{abstract}
The objective of this research is to analyze the health policy structuring process in an Argentinean province during the thirties and the beginning of the forties, showing the importance of this period in the production of changes in state health management in the interior provinces of the country. In order to do so, this paper studies the construction of the health policies and the new state capabilities that were brought into action surrounding public health in the province of Córdoba.
\end{abstract}

KEY WORDS: Health. Social Policies. Interventionism. Córdoba. Argentina.

* Lic. en Historia, Becaria del CONICET, Centro de Estudios Históricos «Prof. Carlos S. A. Segreti» Unidad Asociada a CONICET; Profesora Asistente de la Universidad Nacional de Córdoba. ortizbergia.mj@gmail.com 


\section{INTRODUCCIÓN}

La historiografía argentina y latinoamericana ha prestado una atención preferente al estudio de los procesos de salud/enfermedad en las tres últimas décadas, revelando su fructífero potencial para iluminar las complejas tramas de lo social. Al respecto, Diego Armus ha caracterizado el estado actual de estos estudios a partir de tres abordajes analíticos: una renovada historia de la medicina, una historia sociocultural de las enfermedades y una historia de la salud pública. Esta última especialmente volcada a la investigación del poder, la política, el Estado, la profesión médica y los cambios en las instituciones de salud en el marco de las estructuras económicas, sociales y políticas en las cuales se conformaron. ${ }^{1}$

Desde esta última línea de estudio, los investigadores argentinos han multiplicado sus abordajes sobre el complejo proceso de estructuración de las políticas sanitarias. Estos trabajos en general se insertan en un contexto temporal enmarcado por los procesos de construcción de la llamada Argentina moderna, término bajo el cual pueden sintetizarse los procesos de cambio estructural que atravesó el país entre finales del siglo XIX y comienzos del XX, como la consolidación de un mercado capitalista, un orden estatal y la modernización de las relaciones sociales producto de los intensos procesos de inmigración, crecimiento poblacional y urbanización. Mediante esas investigaciones la historiografía argentina ha puesto en evidencia cómo «el estallido del conflicto social» y, en especial, la movilización obrera del período de entre siglos, interpelaron a las dirigencias políticas, quienes paulatinamente arbitraron innovaciones institucionales tendientes a resolver la llamada «cuestión social». ${ }^{2}$ En ese contexto, las problemáticas sanitarias fueron incluidas en la agenda pública producto de la preocupación de los reformistas sociales, más específicamente de los médicos higienistas. ${ }^{3}$

1 ARmus, D. (2007), La ciudad impura. Salud, tuberculosis y cultura en Buenos Aires, 1870-1950, Buenos Aires, Edhasa, p. 17.

2 SuRIANO, J, (2000), Introducción: una aproximación a la definición de la cuestión social en Argentina. En SuRIANO, J. (comp.), La cuestión social en Argentina 1870-1943, Buenos Aires, La Colmena, 19.

3 ZimMERMANN, E. A. (1995), Los liberales reformistas. La cuestión social en la Argentina 1890-1916, Buenos Aires, Sudamericana, Universidad San Andrés; GonZÁLEZ LEANDRI, R. (2004), El Consejo Nacional de Higiene y la consolidación de una elite profesional al servicio del Estado. Argentina, 1880-1900. Anuario de Estudios Americanos, LXI (2), 571-593; ARMUS, D. (2000), El descubrimiento de la enfermedad como problema social. En LOBATO, M. (dir.), Nueva Historia Argentina. El progreso, la modernización y sus límites (1880-1916), Buenos Aires, Sudamericana, pp. 507-551. 
Asimismo, la mayoría de esos estudios se han dedicado a la indagación de la ciudad de Buenos Aires y de su periferia, centro político y económico del país, mostrando que en ese contexto la brecha entre las expectativas de reforma social y la generación de un equipamiento sanitario se acortó más tempranamente producto del intensivo accionar del Estado federal, municipal y de las asociaciones privadas de mutualismo y de beneficencia. Durante el período de entreguerras, dada la oferta sanitaria existente, se produjeron intensos debates sobre la necesidad de regular y reorganizar ese sistema y se generó una mayor intervención estatal orientada a ampliar los servicios de salud para integrar a nuevos sectores sociales. ${ }^{4}$

Sin embargo, esa trayectoria de los servicios sanitarios en la ciudad porteña da cuenta escasamente de los procesos desarrollados en el interior del país, en donde los gobiernos locales tuvieron mayores dificultades para construir instrumentos eficaces a la hora de promover el mejoramiento de las condiciones sanitarias de sus jurisdicciones. En este sentido, es necesario reconocer que, en un país de fuertes asimetrías regionales, la elaboración de intervenciones sanitarias que mejoraran las condiciones de vida de la población se desplegó a ritmos diversos y desiguales. Desde esa perspectiva, María Silvia Di Liscia ha demostrado que en el Territorio Nacional de La Pampa recién durante los años treinta del siglo XX se produjo un despliegue más intensivo de las intervenciones estatales, en donde la prioridad gubernamental fue expandir los establecimientos de salud sobre espacios rurales y localidades con escasos dispositivos de atención y de profesionales de la salud. ${ }^{5}$

Con la finalidad de profundizar en la discusión sobre esos diversos ritmos en los que se desplegó el proceso de construcción de las políticas sanitarias en

4 Garnino, M. I. y Persello, A. V. (1988), La reformulación del mercado de atención médica. Buenos Aires, 1920-1940. Anuario de Rosario, 13, 343-367; BelmartinO, S. (2005), La atención médica argentina en el siglo XX. Instituciones y procesos, Buenos Aires, Siglo XXI Editores.

5 Di Liscia, M. S. (2007), Dificultades y Desvelos de un Estado interventor. Instituciones, salud y sociedad en el Interior Argentino. La Pampa, 1930-1946. Anuario IEHS, 22, 93123. Pero las investigaciones de Di Liscia no son las únicas que evidencian diferentes periodizaciones en la construcción sanitaria estatal en la Argentina. Al respecto, son importantes las investigaciones realizadas por Susana Belmartino y, más recientemente, por Susana Piazzesi sobre las políticas sanitarias en Santa Fe, estudios que revelan el intenso proceso de elaboración de políticas sanitarias en esa jurisdicción durante la segunda mitad de la década del treinta. Belmartino, S. (2007), Coyuntura crítica y cambio institucional en salud: Argentina en los años '40. Salud Colectiva, 3(2), 177-202; PIAZZESI, S. (2009), Conservadores en Provincia. El iriondismo santafecino 1937-1943. Santa Fe, Universidad Nacional del Litoral. 
la Argentina, en este artículo nos hemos propuesto avanzar en el estudio de una provincia del interior del país que muestra algunas similitudes con el caso pampeano, la provincia de Córdoba. En ese sentido, el análisis de las políticas sanitarias cordobesas muestra cómo incipientemente a partir de los años veinte, ${ }^{6}$ pero más claramente en la coyuntura abierta por la crisis económica mundial, se intensificaron y consolidaron las intervenciones estatales en el campo de la salud. En la provincia ese proceso se expresó a través de la expansión de la infraestructura sanitaria, de la mayor intensidad de las políticas preventivas de salud y de la ampliación de las capacidades de los aparatos públicos para intervenir en la sociedad. Esos cambios pusieron en evidencia indicios de la estructuración de un Estado social que permitió paulatinamente alcanzar un mayor grado de desmercantilización de las necesidades de individuos y familias y, por tanto, permitir independizar sus demandas de acceso a la salud de las condiciones impuestas por el mercado. ${ }^{7}$

Es necesario señalar que la jurisdicción cordobesa en esa coyuntura contaba con una población estimada en un millón y medio de habitantes distribuida en una extensa superficie territorial superior a los $160.000 \mathrm{~km}^{2}{ }^{8}$ Además, más allá de su peso territorial y poblacional, constituía para la época una muestra de la misma complejidad que encerraba un país de fuerte diversidad regional como la Argentina. La provincia tenía una doble inserción socioeconómica que complejizaba el marco en el cual se desarrollaban las intervenciones de los gobiernos locales. Por una parte, su zona sudeste se caracterizaba por un importante crecimiento económico producto de su incorporación al modelo agrario exportador de la pampa argentina; por otra parte, la zona noroeste del territorio provincial, centro económico del período colonial e independiente y

6 CArbonetti, A. (2005), La conformación del sistema sanitario de la Argentina. El caso de la Provincia de Córdoba, 1880-1926. Dynamis, 25, 87-116; MoreYrA, B. I. (2009), Cuestión social y políticas sociales en la Argentina. La modernidad periférica. Córdoba, 19001930, Bernal, Universidad Nacional de Quilmes.

7 Esping-Andersen G. (1990), The Three Worlds of Welfare Capitalism, New Jersey, Princeton University Press, 37.

8 Para el período en que estamos trabajando es difícil calcular su población dada la falta de estadísticas fidedignas a lo largo del período 1914-1947. De todos modos, las oficinas públicas calculaban que en 1935 la población total de la provincia era de 1.235 .398 y de la capital provincial de 268.277. Finalmente, en el censo nacional de 1947 la población de la provincia se fijó en 1.491.987 habitantes y la de la capital provincial en 386.828 habitantes. Dirección General de Estadísticas de la Provincia de Córdoba, Anuario estadístico, año 1935, Talleres Gráficos de «Comercio y Tribunales», Córdoba, 1938; Dirección Nacional de Servicio Estadístico, IV Censo General de la Nación, Buenos Aires, Vol. 1-2, p. 172. 
de más antiguo poblamiento, atravesaba durante el primer tercio del siglo XX un proceso de fuerte depresión económica y de agotamiento de los modos de vida preexistentes al quedar marginada de los procesos socioeconómicos que se vivían en la zona sudeste. En ese sentido, Córdoba era un territorio en el que convivían la Argentina moderna, la zona pampeana, de desarrollo económico vertiginoso y de arribo de inmigración europea, y la Argentina del interior, caracterizada por sus dificultades para insertarse en el proceso de crecimiento que protagonizaba la primera.

En ese contexto, el eje de esta investigación es el de analizar los cambios producidos en las políticas públicas de salud de la provincia de Córdoba, reconstruyendo las tendencias en la conformación de las intervenciones estatales, las rupturas y las continuidades, los obstáculos y los condicionamientos y los factores causales que incidieron en el despliegue de políticas sanitarias en los años previos a la emergencia del peronismo en la Argentina. Para ello, indagamos en la trayectoria del organismo estatal dedicado a la atención de la salud, el Consejo Provincial de Higiene (en adelante $\mathrm{CPH}$ ), en sus objetivos, en el contenido y orientación de sus intervenciones y en el desarrollo de sus capacidades institucionales, humanas y materiales. Respecto a esto último, en este trabajo adoptamos el concepto de «capacidades estatales» para aprehender las diferentes y las variables «aptitudes de las instancias de gobierno para obtener resultados $\gg \vartheta^{9}$ a través de sus intervenciones. Más precisamente, en esta investigación nos limitaremos a revisar aquellos aspectos que se relacionan con los contenidos administrativos de las capacidades estatales, a dimensiones tecno-burocráticas tales como los recursos humanos, la organización interna y las bases financieras que condicionan las posibilidades efectivas de los aparatos estatales para implementar políticas públicas. ${ }^{10}$

La investigación se estructuró en tres apartados. En el primero de ellos nos abocamos a proporcionar un panorama general sobre las características del sistema sanitario disponible a comienzos de los años treinta en la provincia de Córdoba, destacando los objetivos institucionales y las intervenciones que

9 RepetTo F. y ANDRENACCi L. (2006), Ciudadanía y política pública: dilemas de reconstrucción de la política social argentina. En ANDRENACCI, L. (comp.) (2006), Problemas de política social en la Argentina contemporánea, UNGS, Prometeo, 313.

10 Conceptualmente podemos distinguir entre dos dimensiones de las capacidades estatales, las administrativas y las políticas, estas últimas remiten a las posibilidades estatales para imponer reglas y su habilidad para procesar las demandas sociales, políticas y económicas provenientes de la sociedad. Alonso, G. V. (ed.) (2007), Capacidades estatales, instituciones y política social, Buenos Aires, Prometeo, pp. 18-19. 
desplegaba el CPH. En una segunda parte, nos propusimos estudiar las modificaciones y permanencias en las funciones del organismo sanitario provincial, en el contenido y orientación de su accionar y en el desarrollo de sus capacidades institucionales a lo largo de la década del treinta y comienzos de la del cuarenta (1930-1943). Finalmente, el último apartado está dedicado a analizar los cambios contextuales, políticos, sociales y culturales, que impulsaron y condicionaron la trayectoria del aparato estatal a lo largo de esos años en la provincia de Córdoba.

\section{LAS POLÍTICAS SANITARIAS EN LAS PRIMERAS DÉCADAS DEL SIGLO XX}

A lo largo de las tres primeras décadas del siglo XX existieron diferentes actores participando en el campo de la salud en la provincia de Córdoba municipios, Nación, provincia, sociedades de beneficencia, sanatorios privados y médicos particulares, a lo que no sería excepcional agregar a los idóneos en el arte de curar. Esta diversidad de actores conformaba un sistema heterogéneo, disperso y escaso en relación a las necesidades de su población. Así pues, la infraestructura existente en 1914, al margen de la capital provincial, consistía en sólo siete entidades hospitalarias, públicas y de beneficencia, en todo el territorio provincial. ${ }^{11}$ Para 1930, a éstas se sumaron otras siete, junto a dispensarios en las zonas menos pobladas, pero la norma fue la insuficiente penetración de la infraestructura sanitaria, en especial en las zonas más marginales socioeconómicamente como el noroeste cordobés. ${ }^{12}$ En el caso de la ciudad capital de la provincia, para 1930, era una urbe de cerca de 300.000 habitantes con sólo seis hospitales generales, ${ }^{13}$ dos destinados a la tuberculosis y uno a enfermedades infectocontagiosas. Entonces, hasta los años treinta el sistema sanitario se manifestaba como un deficiente tendido de dispositivos que no se adecuaban a la dispersión geográfica y al volumen de su población.

En ese modelo asistencial, la participación del gobierno provincial se encontraba fuertemente limitada, prácticamente ausente de la administración de la infraestructura sanitaria pública, reduciendo sus actividades a la regulación

11 MOREyra (2009), p. 122.

12 Ibid., p. 130.

13 Entre ellos se contaban el hospital universitario, el hospital de Niños de la Sociedad de Beneficencia, el Español y el Italiano de las sociedades de beneficencia de sendas colectividades y el Hospital San Roque bajo el Patronato del gobierno provincial. Finalmente, existía un Hospital Militar. 
del ejercicio de la medicina y a la intervención esporádica en la atención de las enfermedades infecciosas. También mantenía estrechas relaciones con la actividad privada con una política flexible de asignación de subvenciones, subsidios y obras públicas destinadas a sustentar el accionar de las organizaciones de beneficencia. Prevalecía, de ese modo, un sistema prescindente, predominantemente orientado por una concepción liberal de las políticas sociales, de políticas sanitarias fuertemente condicionadas por perspectivas epidemiológicas y con actuaciones de carácter más defensivo que preventivo.

Hacia mediados de los años veinte se comenzaron a producir incipientes cambios en este esquema a partir de la difusión de dispensarios, la atención directa del problema de la salud y la centralización de todas las instancias administrativas. ${ }^{14}$ No obstante, la imagen que brindaba el sistema sanitario de la provincia en 1930 demuestra que esos cambios no eran más que esbozos de procesos más amplios que se produjeron años después. Para ese año, el gobierno bajo su estricto control contaba en todo el territorio de su jurisdicción con sólo tres dispensarios para niños, tres antituberculosos, un conjunto de establecimientos antivenéreos y un hospital. Asimismo, las actividades específicas del CPH en el campo de la salud se restringían a la regulación de los profesionales de la salud y a la administración de un precario sistema de atención de las enfermedades infectocontagiosas. En el año 1929, la labor del organismo había consistido en la regulación del desempeño de los profesionales de la salud y de sus prácticas ilegales, en la elaboración de informes médicos legales, inspecciones de higiene y de enfermedades infecciosas y a la distribución de vacunas y sueros. ${ }^{15}$ La política social provincial en las tres primeras décadas del siglo XX sólo había sufrido «desplazamientos parciales y coyunturales en una matriz histórica de permanencias.» ${ }^{16}$

\section{LAS POLÍTICAS SANITARIAS EN LOS AÑOS TREINTA}

Iniciada la década del treinta dos procesos importantes caracterizaron la política sanitaria provincial. Por un lado, es factible identificar un proceso de ampliación de las intervenciones estatales en el campo de la salud, a través de cambios en la infraestructura sanitaria y de un despliegue más intensivo de políticas preventivas. Por otro lado, se produjo una redefinición de las prioridades gubernamentales, crecimiento del aparato público y de las capacidades

14 CARbonetTi (2005).

15 La Voz del Interior, 4/4/30, p. 11.

16 Moreyra (2009), p. 285. 
estatales para intervenir sobre las problemáticas sanitarias, procesos que se desplegaron en torno al Consejo Provincial de Higiene.

\section{El sistema sanitario provincial en transformación}

Un primer aspecto de los procesos antes mencionados se visualiza en los guarismos transcritos en la Tabla 1 que muestran el crecimiento constante de la infraestructura sanitaria de la provincia entre 1933 y 1943. Este aumento de la infraestructura implicó un quiebre en las políticas sanitarias que habían impulsado los gobiernos locales a lo largo de las primeras décadas del siglo XX, generando que en pocos años el Estado cordobés se convirtiera en una importante organización en la prestación de atención médica para la población aún en los espacios más distantes del territorio.

TABLA 1. INFRAESTRUCTURA SANITARIA PÚBLICA DE LA PROVINCIA DE CÓRDOBA, SEGÚN LAS LEYES DE PRESUPUESTO, 1932-194317

\begin{tabular}{|l|c|c|c|c|c|c|c|c|}
\hline & 1932 & 1933 & 1934 & 1936 & 1939 & 1941 & 1942 & $\mathbf{1 9 4 3}$ \\
\hline $\begin{array}{l}\text { Gotas de Leche-Dispensarios de } \\
\text { Lactantes }\end{array}$ & 5 & 5 & 7 & 7 & 7 & 8 & 8 & $\mathbf{8}$ \\
\hline Dispensarios Antituberculosos & 4 & 4 & 5 & 5 & 5 & 5 & 5 & $\mathbf{5}$ \\
\hline Dispensarios antivenéreos & 14 & 12 & 9 & 9 & 8 & 10 & 10 & $\mathbf{9}$ \\
\hline Pabellón de Leprosos & & & & 1 & 1 & 1 & 1 & $\mathbf{1}$ \\
\hline $\begin{array}{l}\text { Dispensarios de Profilaxis General, } \\
\text { Primeros Auxilios y Atención Médica }\end{array}$ & & 11 & 22 & 41 & 48 & 55 & 56 & $\mathbf{5 7}$ \\
\hline Hospitales & 1 & 1 & 1 & 1 & 2 & 5 & 9 & $\mathbf{8}$ \\
\hline Maternidades & & & & 2 & 1 & 1 & 1 & $\mathbf{2}$ \\
\hline Estación Sanitaria del Noroeste & & & & & 1 & 1 & 1 & $\mathbf{1}$ \\
\hline Destacamentos Sanitarios & & & & & 6 & 4 & 4 & $\mathbf{7}$ \\
\hline Sanatorio de Previsión Social & & & & & 1 & 1 & 1 & $\mathbf{1}$ \\
\hline Sanatorio Psiquiátrico Provincial & & & & & & & 1 & $\mathbf{1}$ \\
\hline
\end{tabular}

Elaboración propia. Fuente: Compilación de Leyes de la Provincia de Córdoba, 1932-1942; Archivo de Gobierno de la Provincia de Córdoba, Ministerio de Gobierno, año 1944, t. 4, fs. 61r-87r.

*Extraído de una nómina de personal del año 1943.

17 El cuadro tiene sólo un carácter referencial dado que no necesariamente los datos contenidos en las leyes de presupuesto pueden ser tomados como fidedignos de los establecimientos creados en los años asignados. 
Un aspecto que nos parece importante destacar es que los establecimientos destinados a la atención de enfermedades sociales como las afecciones venéreas y la tuberculosis tuvieron un ralentizado crecimiento a lo largo de esos años respecto a los dedicados a la atención asistencial de la población, dispensarios y hospitales, una cuestión que remite a cambios en las preocupaciones sanitarias centrales de las autoridades públicas. En ese sentido, las finalidades que presidieron la creación de los nuevos establecimientos distaron de responder predominantemente a las inquietudes por las enfermedades resultado de procesos infecciosos, de los cuales la sociedad debía defenderse a través de mecanismos de control, aislamiento, desinfección y represión. Estas innovaciones, en cambio, eran el resultado de una preocupación por asegurar el acceso a la atención sanitaria asistencial de la población. El gobernador, autoridad máxima de la provincia, explicaba que los dispensarios que se estaban construyendo respondían a «una nueva orientación y finalidad más amplia, dada la índole de sus funciones. El carácter de sus servicios se extiende a la asistencia médica general de acuerdo a las necesidades de la población. Instalados en las regiones más pobres de la Provincia prestan incalculables beneficios a los vecindarios, alejados hoy del peligro del curanderismo y descongestionando evidentemente los hospitales de la ciudad. ${ }^{18}$ En ese sentido, este tipo de intervenciones se desplegaron en especial en la denominada campaña provincial, pueblos y ciudades rodeadas de amplios espacios rurales que generaban su aislamiento de la prestación de los servicios públicos sanitarios.

Sobre esto último es importante destacar que la construcción de dispensarios de profilaxis general y, aunque más lentamente, de hospitales regionales, permitió no sólo dar satisfacción a las necesidades inmediatas de pueblos y ciudades, sino también descentralizar los servicios de salud de la capital provincial que se encontraban sobresaturados por la concurrencia de enfermos del resto de la jurisdicción. Pero eso no implicaba que no existieran preocupaciones similares para con la población de la ciudad de Córdoba, capital del territorio, sino que la provincia delegó su atención en otras jurisdicciones. En la Capital, estas iniciativas, tendientes a descentralizar la atención sanitaria y a mejorar sus condiciones de acceso, fueron protagonizadas por el municipio local. Entre 1932 y 1942 se crearon nueve dispensarios seccionales en las distintas barriadas de la ciudad, descentralizando los servicios de salud. Desde el municipio se destacaba la finalidad de este tipo de establecimientos: «sus servicios tienen un carácter y una finalidad distinta: es la medicina de ur-

18 Secretaría Técnica Parlamentaria, Cámara de Diputados, Mensajes a la Legislatura acerca del Estado de la Provincia. Gobernador Pedro J. Frías, 1933-1935, Córdoba, 1992, p. 85. 
gencia y la clínica general, la que más solicitan los barrios obreros a donde la carencia de recursos repercute en el organismo, privándole de los elementos indispensables que aseguran el crecimiento y proveen a su defensa.. ${ }^{19}$ Entonces, la provincia desarrolló su accionar en las zonas semiurbanas y dejó en manos del municipio capitalino la ampliación de los servicios médicos primarios de su población. La lógica en la base de esa política fue la existencia de otros participantes que podían subsanar la escasa actividad provincial, permitiendo a las autoridades provinciales dedicarse a expandir los servicios de salud en aquellos lugares donde los dispositivos sanitarios eran escasos o nulos.

TABla 2. Hospital y DisPensarios De ProfilaXis GeNERAL EN 1943

\begin{tabular}{|c|c|c|}
\hline Tipo de establecimiento & Número & Localización \\
\hline Hospitales Regionales & 8 & $\begin{array}{l}\text { Deán Funes, Leones, Cruz Alta, General Cabrera, } \\
\text { Arroyito, Pozo del Molle, Villa de María, Corral de } \\
\text { Bustos }\end{array}$ \\
\hline $\begin{array}{l}\text { Dispensarios de Profilaxis } \\
\text { General, Primeros Auxilios y } \\
\text { Atención Médica }\end{array}$ & 57 & $\begin{array}{l}\text { Ambul, Athos Pampa, Balnearia, Buchardo, Canals, } \\
\text { Capilla de los Remedios, Carrilobo, Ciénaga del } \\
\text { Coro, Despeñaderos, Devoto, El Arañado, General } \\
\text { Levalle, Gigena, Hernando, Huerta Grande, Jesús } \\
\text { María, Jovita, La Calera, La Carlota, La Cruz, La } \\
\text { Laguna, La Paz, La Playosa, Laborde, Laguna Lar- } \\
\text { ga, Las Arrias, Las Perdices, Las Varillas, Los Rear- } \\
\text { tes, Mina Clavero, Moldes, Noetinger, Obispo Trejo, } \\
\text { Pampa de Achala, Quilino, Reducción, Río Primero, } \\
\text { Río Segundo, Río Tercero, Salsacate, San Agustín, } \\
\text { San Francisco del Chañar, San Javier, Santiago } \\
\text { Temple, San Vicente, Santa Rosa de Río Primero, } \\
\text { Serrezuela, Soto, Tancacha, Tránsito, Tulumba, } \\
\text { Ucacha, Unquillo, Villa Ascasubi, Villa Concepción } \\
\text { del Tío, Villa General Mitre, Villa Valeria. }\end{array}$ \\
\hline
\end{tabular}

Fuente: Archivo de Gobierno de la Provincia de Córdoba, Ministerio de Gobierno, año 1944, t. 4, fs. $61 \mathrm{r}-87 \mathrm{r}$.

Recapitulando, en esos años treinta el gobierno modificó el lugar que ocupaba en el campo de la salud pública a través de la creación de numerosa infraestructura sanitaria. Ese proceso fue acompañado por nuevas concepciones y objetivos socio-políticos en torno al problema de la salud, en especial,

19 Los Principios, 9/5/32, p. 2. 
orientados a favorecer el acceso a prestaciones de carácter asistencial a las poblaciones que carecían de servicios sanitarios regulares y gratuitos. En ese sentido, existieron procesos similares con los evidenciados por Di Liscia para el Territorio Nacional de La Pampa, en donde el accionar estatal intentó penetrar los territorios rurales y semirurales con políticas sanitarias que pusieran al alcance de la población los avances médicos. ${ }^{20}$ Pero, como en el caso de La Pampa, en la provincia de Córdoba existen otros elementos que también confirman esta consolidación del Estado provincial en el campo de la salud, como las campañas sanitarias.

En esos años, afecciones como la difteria, la fiebre tifoidea, la peste bubónica, la viruela, el paludismo y el tracoma, constituyeron nudos centrales de las preocupaciones sanitarias de las autoridades. Dos instrumentos se desplegaron en su tratamiento, por un lado, políticas preventivas como la distribución de vacunas y la realización de campañas educativas de salud, por el otro, las intervenciones sobre los brotes epidémicos que sobrevinieron a lo largo de la década.

En lo que hace al primero de estos instrumentos existió un pronunciado incremento en las acciones del CPH destinadas a impulsar campañas preventivas de salud, consistentes en vacunaciones y en la difusión de preceptos higiénicos. Además de la vacunación antivariólica, a partir de 1932 se estableció la obligatoriedad y gratuidad de las antitíficas y antidiftéricas en las escuelas públicas y privadas. El aumento de las capacidades del CPH para realizar este tipo de intervenciones se puso en evidencia en 1936, producto de una serie brotes de viruela en el país, episodios que incentivaron una intensa campaña de vacunación y revacunación. Hacia finales de ese año se habían distribuido 359.780 vacunas comprendiendo no sólo a los escolares, sino gratuitamente a empleados públicos provinciales, municipales, nacionales, de sociedades particulares y a la población que así lo requiriera. El mismo presidente del Departamento Nacional de Higiene, ente nacional dedicado a la política sanitaria, destacaba sobre el organismo local «Indudablemente, le cabe a Córdoba y a sus autoridades sanitarias el haber sido la primera provincia que atacó con mayor energía, eficacia e intensidad la tarea de vacunación y revacunación de la mayor parte de su población. Esta campaña realizada en el período precoz, ha dado grandes resultados y el Consejo Nacional no puede sino estar contento de hacerlo constar así»». ${ }^{21}$

Asimismo, otra faceta de este interés por las medidas de carácter preventivo fueron las campañas de difusión de preceptos higiénicos, tales como la

\footnotetext{
20 Di LisCIA (2007).

$21 \quad$ La Voz del Interior, 19/12/36, p. 6.
} 
educación antivenérea, el combate de la mosca asociada a la propagación de enfermedades como la poliomielitis y la difusión de consejos propios de la Puericultura sobre la mejor forma de cuidar a los niños en los dispensarios de lactantes y en la «Semana del Nene». Incluso, en 1937 el CPH organizó una Exposición de Higiene destinada a poner en evidencia los logros alcanzados, los avances científicos y los nuevos objetivos sanitarios bajo jurisdicción estatal. La misma contó con la participación de las reparticiones provinciales, municipales, nacionales, asociaciones deportivas, industriales, casas comerciales y compañías de servicios públicos. Más de 50.000 personas visitaron la muestra a los que se sumaron los escolares. ${ }^{22}$

Un tercer campo en el que se desplegaron las actividades del $\mathrm{CPH}$ fue en la acción profiláctica en casos de brotes epidémicos. Sin embargo, en estos episodios en general las intervenciones fueron de carácter más bien tardío y reactivo, caracterizándose por sus dificultades para dar respuesta a los episodios de enfermedades infecciosas.

En el caso de la provincia, uno de los azotes permanentes del período fue la peste bubónica favorecida por la importante producción agrícola que generaba la proliferación de roedores cerca del hábitat humano. La política de control de la peste consistía en el saneamiento permanente y la desratización. Un accionar de este tipo habían impulsado las autoridades a comienzos de la década a través de una campaña de desratización aplicando nuevos métodos de exterminio que consistían en la construcción de barreras dentro de las cuales se utilizaban perros ratoneros, máquinas fumigadoras y cartuchos asfixiantes. El éxito de esta política preventiva fue la inexistencia de casos mortales de bubónica entre 1934 y 1935 . Pero para 1940 la falta de continuidad de estas medidas incidió en un nuevo brote de peste bubónica con el resultado de 103 enfermos en el territorio provincial y la existencia de casos durante los dos años consecutivos. ${ }^{23} \mathrm{La}$ inadecuación de los mecanismos de atención se puso en evidencia en los pedidos de recursos extraordinarios para adquirir los insumos necesarios para la campaña de profilaxis y el tratamiento de los enfermos, como la contratación de profesionales y peones, la compra de medicamentos, camiones sanitarios y carpa-hospital. Esta coyuntura mostró las dificul-

22 Secretaría Técnica Parlamentaria, Cámara de Diputados, Mensajes a la Legislatura acerca del Estado de la Provincia. Gobernador Amadeo Sabattini, Córdoba, 1992, p. 96.

23 A partir de ese año se produjo una paulatina disminución de los casos, 52 en 1941 de los cuales sólo 27 fueron mortales y 30 en 1942. Mensaje a la Legislatura del Gobernador de la Provincia Santiago del Castillo, año 1941, p. 17; Archivo de Gobierno de la Provincia de Córdoba (en adelante AGPC), Ministerio de Gobierno, año 1942, t. 43, f. 240r. 
tades del gobierno local para consolidar una estructura permanente de saneamiento y control, en parte producto de su alto costo económico y de su escasa relevancia pública fuera de los períodos en que la enfermedad cobraba virulencia.

Una trayectoria accidentada similar sufrió el tratamiento del paludismo. En 1933 se produjo un brote en plena capital provincial durante la cual el CPH se dedicó a limpiar el cauce de los ríos y a distribuir quinina entre 2.000 enfermos o sospechosos de serlo. Al igual que en el caso de la peste bubónica, en 1936, un grave brote de paludismo en el norte de la provincia requirió la sanción de fondos extraordinarios para atender la problemática, nuevamente distribución de quinina, limpieza de cauces y camiones sanitarios. De todos modos, este episodio implicó una novedad respecto a los precedentes, dio lugar a la generación de una estructura permanente de atención de las enfermedades endémicas del norte cordobés como el paludismo, la enfermedad de Chagas, el tracoma y la brucelosis, un establecimiento sanitario dedicado a la lucha continua contra esas afecciones.

En síntesis, a lo largo de estos años se produjo una reforma estructural en la participación del gobierno provincial en el campo de la salud. El eje de esas modificaciones fue la expansión de la infraestructura a disposición de la población y una renovada orientación de la misma en especial tendiente a atender más sistemáticamente a la población rural de la provincia. Este cambio fue acompañado por el despliegue más intensivo de políticas de carácter preventivo como las campañas de vacunación y de educación sanitaria. Estos dos aspectos mostrarían las innovaciones institucionales más destacadas. En contraste, las intervenciones destinadas a atender las enfermedades endémicas en los ámbitos rurales mostraron los límites de esos procesos, a través de la necesidad de realizar inversiones extraordinarias en condiciones críticas y la desigual atención dada a través del tiempo a las políticas de saneamiento.

\section{Los cambios en las capacidades estatales: el Consejo Provincial de Higiene}

Este apartado está dedicado a investigar la conformación de las capacidades del CPH para intervenir en las problemáticas sanitarias de los cordobeses, para regular las relaciones sociales, penetrar e imponer sus directivas sobre el conjunto de la sociedad. Para ello nos hemos propuesto revisar qué modificaciones se produjeron en la estructura del aparato estatal, estudiando los cambios en su organigrama interno y en sus recursos humanos y materiales.

Uno de los primeros indicadores de los procesos de cambio institucional que se produjeron en los años treinta es el de la ampliación de los objetivos 
del organismo. En 1930 el funcionario a cargo del CPH, el Dr. Vázquez Amenabar, reseñaba que las tareas del mismo habían sido las de atender a la profilaxis de las enfermedades epidémicas, la regulación de los profesionales de la salud, los informes médicos legales y la distribución de sueros y vacunas. Sin embargo, la expansión de la infraestructura sanitaria, la mayor expectativa en el mejoramiento en las condiciones de acceso a la medicina, la misma ampliación del concepto de salud, no sólo como ausencia de enfermedad, sino como una condición de bienestar integral, incidieron en el crecimiento de los objetivos institucionales del CPH. En 1936, el gobierno al presentar un proyecto de creación del Departamento Provincial de Higiene, incluyó como finalidades del organismo no sólo las funciones tradicionales de profilaxis y reglamentación, sino también: el de ejercer la superintendencia sobre todas las instituciones y servicios sanitarios que dependieran directamente del gobierno y la inspección y la vigilancia de aquellos que recibieran financiamiento de la provincia. Además, debía organizar comisiones asesoras técnicas para el estudio de los medios defensivos frente a las enfermedades, dirigir y orientar el deporte y propender al mejoramiento de la vivienda y de la alimentación popular. ${ }^{24}$ El propósito del mismo era la estructuración de un organismo «con amplias facultades [en] el extenso radio de acción, confiándole la defensa integral de la sociedad frente a los daños que puedan originarle las enfermedades, la mala vivienda, la alimentación deficiente, el trabajo insalubre y todo otro factor negativo respecto a su salud y progreso. $\rangle^{25}$ En realidad, este proyecto buscaba sancionar ex post facto algunas de las nuevas funciones que se habían ido incorporando en la agenda de gobierno con la ampliación de la infraestructura y la educación sanitaria, las políticas de salud de carácter preventivo y las nuevas injerencias como el control bromatológico. Finalmente, estas modificaciones en los objetivos institucionales del organismo fueron acompañadas también por cambios en su organigrama interno, con el crecimiento de sus secciones y dependencias.

En lo que hace a la ampliación de su estructura, ya en 1930 se abrió un espacio de producción de vacunas y sueros aunque el mismo carecía de la infraestructura y de los recursos necesarios para desarrollar eficazmente sus tareas. ${ }^{26}$ Recién en 1932 se creó el Instituto de Higiene, repartición destinada a la producción de vacunas y sueros, análisis químicos, bacteriológicos y bromatológicos. La ampliación de las necesidades de análisis, estudios e investigaciones llevó a la complejización de esta sección que devino en la de

24 Diario de Sesiones de la Cámara de Diputados de la Provincia de Córdoba, 18/8/36, p. 824. 
Laboratorios. Además, se creó un área de bromatología destinada al control de la calidad de los alimentos y, finalmente, en 1940, se organizó una Farmacia con la finalidad de producir los insumos médicos de las reparticiones provinciales. Estas innovaciones tuvieron como propósito construir una mayor capacidad de intervención y, a su vez, de autonomía para la política sanitaria provincial.

TABla 3. ORGANIZACiÓN Y PERSONAL DEL CONSEJo PROVINCIAL DE HigIENE, 1932-1943

\begin{tabular}{|c|c|c|c|c|c|c|c|c|}
\hline \multirow{3}{*}{ Secciones centrales } & \multicolumn{8}{|c|}{ Personal de la repartición } \\
\hline & \multicolumn{7}{|c|}{ Según Leyes de Presupuesto* } & \multirow{2}{*}{$\begin{array}{l}\text { Personal Efec- } \\
\text { tivo en } 1943^{* *}\end{array}$} \\
\hline & 1932 & 1933 & 1934 & 1936 & 1939 & 1941 & 1942 & \\
\hline Dirección & 19 & 19 & 19 & 19 & 8 & 8 & 8 & 8 \\
\hline Sección Médico Escolar & 9 & 9 & 9 & 13 & 13 & & & \\
\hline $\begin{array}{l}\text { Instituto de Higiene (1932) } \\
\text { Laboratorio y Gabinetes } \\
\text { (1939) }\end{array}$ & 8 & 12 & 12 & 13 & 13 & 2 & 2 & 2 \\
\hline Subsección Química & & & & & & 3 & 3 & 3 \\
\hline $\begin{array}{l}\text { Subsección Bromatología y } \\
\text { Farmacia Central }\end{array}$ & & & & & & 6 & 6 & 6 \\
\hline Bacteriología y Serología & & & & & & 5 & 5 & 5 \\
\hline Instituto Antirrábico & 2 & 2 & 2 & 2 & & 3 & 3 & 3 \\
\hline Profilaxis General e Higiene & & & & & 10 & 10 & 15 & 16 \\
\hline Asistencia Médico-Social & & & & & 3 & 3 & 3 & 3 \\
\hline Asuntos Legales & & & & & 2 & 2 & 2 & 2 \\
\hline Inspección de Farmacias & & & & & 7 & 6 & 6 & 6 \\
\hline \begin{tabular}{|l} 
Total \\
\end{tabular} & 40 & 42 & 42 & 47 & 56 & 48 & 53 & 54 \\
\hline $\begin{array}{l}\text { Total sin sección Médico esco- } \\
\text { lar*** }\end{array}$ & 31 & 33 & 33 & 34 & 43 & 48 & 53 & \\
\hline
\end{tabular}

* Compilación de Leyes de la Provincia de Córdoba, 1932-1942.

** AGPC, Ministerio de Gobierno, año 1944, t. 4, fs. 61r-87r.

*** La sección Médico Escolar pasó a formar parte del Consejo General de Educación a partir del presupuesto de 1941.

En la anterior tabla se puede constatar este proceso de ampliación de las secciones burocráticas y técnicas del $\mathrm{CPH}$ y de los empleados en cada una de ellas. Como se puede observar existió a lo largo de esos años una complejiza- 
ción de su organigrama, tendiente a alcanzar una mejor distribución de las labores, delimitando las tareas, permitiendo alcanzar una mayor especialización y experiencia en las actividades que desplegaba la repartición. ${ }^{27}$ Los espacios que adquirieron una mayor centralidad fueron las áreas de laboratorios y de producción de insumos médicos destinados a dotar al organismo de mejores condiciones para el desarrollo de sus intervenciones. Asimismo, la mayor división de las tareas permitió la optimización de la labor administrativa en cada una de las secciones.

Acompañando este proceso, a lo largo del período los recursos públicos invertidos en el organismo fueron creciendo paulatinamente. Incluso, en la coyuntura de los primeros años de la década, afectada por la crisis económica mundial, la tendencia a que los montos destinados a salud superaran en términos relativos el gasto provincial total fue constante.

TABLA 4. VARIACIÓN RELATIVA DEL GASTO TOTAL Y EL GASTO EN SALUD PROVINCIAL. $(1930=100), 1930-1942$

\begin{tabular}{|c|c|c|}
\hline & Salud & Gasto total \\
\hline 1930 & 100 & 100 \\
\hline 1931 & 103 & 94 \\
\hline 1932 & 102 & 86 \\
\hline 1933 & 99 & 107 \\
\hline 1934 & 102 & 87 \\
\hline 1935 & 104 & 111 \\
\hline 1936 & 109 & 84 \\
\hline 1937 & 113 & 87 \\
\hline 1938 & 133 & 96 \\
\hline 1939 & 164 & 103 \\
\hline 1940 & 174 & 106 \\
\hline 1941 & 179 & 107 \\
\hline 1942 & 184 & 115 \\
\hline
\end{tabular}

Fuente: Anuario Estadístico de la Provincia de Córdoba, años 1930-1942.

De todos modos, la participación relativa de la salud en las inversiones estatales fue poco significativa, pasando del 4 al $5 \%$ del presupuesto de gas-

27 AGPC, Ministerio de Gobierno, año 1940, t. 45, f. 210 r. 
tos. ${ }^{28}$ El presidente del CPH, Dr. Guillermo Stuckert, se quejaba al ministro de gobierno por esta política de asignación del gasto público. El funcionario destacaba que la inversión de fondos en el organismo no era coherente con las expectativas puestas en el mismo $y$, en términos relativos, era insignificante respecto a otras reparticiones destinadas a la educación y a las obras públicas. ${ }^{29}$ Este escaso protagonismo del organismo dentro de los rubros del presupuesto provincial incidía en su desenvolvimiento. Esto se pone en evidencia en el lento crecimiento de los recursos humanos en relación al incremento significativo de sus actividades. ${ }^{30}$.

Por lo tanto, si bien la trayectoria del CPH a lo largo de los años treinta estuvo marcada por la constante expansión del Estado en el campo de la salud pública, con nuevos objetivos institucionales, la ampliación de la estructura del aparato estatal y su reorganización. Existieron una serie de condicionamientos a estas nuevas funciones del Estado provincial en el campo de la salud pública, siendo uno de los principales la política de asignación del gasto. En ese sentido, la dotación de recursos materiales y humanos fue una permanente fuente de dificultades en la realización de sus actividades, limitando los proyectos de sus funcionarios y la penetración del organismo estatal en el territorio.

\section{UN CONTEXTO HISTÓRICO CON SENTIDO}

Como señalamos anteriormente, existieron un conjunto de procesos sociales, económicos, políticos y culturales que explican las modificaciones reseñadas, una matriz histórica que les dio sentido, impulsó su desarrollo y marcó sus límites.

Un primer elemento que parece insoslayable de revisar es el de las modificaciones producidas en la sociedad, en sus condiciones materiales, sus prácticas y expectativas en relación a los servicios sanitarios brindados por el poder público y cómo éstos impactaron en el diseño de las políticas sociales. En ese sentido, según las investigaciones contemporáneas, a lo largo de los años treinta, se produjeron importantes modificaciones en las condiciones sanita-

28 Leyes de Presupuesto, Compilación de Leyes de la Provincia de Córdoba, 1932-1942.

29 AGPC, Ministerio de Gobierno, año 1942, t. 43, f. 239r.

30 Como ejemplo particular, en 1942, la sección de Asistencia Médico-Social debía llevar el control de los establecimientos sanitarios públicos y las instituciones de beneficencia en toda la provincia con un personal consistente en tres personas -un médico en jefe, un subinspector $\mathrm{y}$ un escribiente. 
rias de la población, que para el caso de la ciudad de Córdoba ha llevado a calificar esos años como propios del desarrollo de una transición epidemiológica, un cambio que supuso la caída de las afecciones infecto-contagiosas, de la mortalidad infantil y un aumento de la esperanza de vida al nacer. ${ }^{31}$ Según Dora Celton existió una disminución de la mortalidad bruta que entre 1930 y 1944 pasó de $16,4 \%$ a $12,0 \%$, con dos períodos de mejoras que fueron entre 1930-1934 y 1940-1944. ${ }^{32}$ Esto fue producto de la disminución de las enfermedades infecciosas y respiratorias, entre las que se encontraban las parasitarias, la gripe o influenza, neumonía y bronquitis para los menores de cinco años y demás enfermedades respiratorias. La contraparte de ese proceso fue un aumento de otras afecciones como el cáncer y las cardiovasculares.

Además, se dieron cambios en los porcentajes de mortalidad según la estructura de edad. Se dio un proceso de disminución de los porcentajes de mortalidad en los menores de 15 años. Entre 1935 y 1945, se calcula que la mortalidad infantil pasó de $199,25 \%$ a $85,25 \%$, con una disminución media anual entre 1925-1935 de 4,45\%o, de 9,55\%o entre $1935-1940$ y de 3,25\% entre 1940-1945. Esa disminución relativa de la mortalidad infantil se relacionó con el decrecimiento de las enfermedades de tipo infeccioso parasitario en la población menor a los 15 años. Ese proceso tuvo como consecuencia un aumento en la esperanza de vida al nacer que en el período intercensal 19141947 pasó de 34,7 a 53,9 años, un aumento de 19 años. De todos modos, las enfermedades infecciosas y respiratorias siguieron teniendo una fuerte incidencia entre las causas de mortalidad general hasta bien entrada la década del cuarenta, pero pasaron del 61,5\% a 37,3\% de la mortalidad bruta entre 1925 y 1947. Esa reducción del nivel de mortalidad fue provocada por una pérdida de importancia de las enfermedades infecto-contagiosas pero con una permanencia de afecciones como la tuberculosis pulmonar y la meningitis.

En lo que se ha dado en denominar como afecciones modernas, en las que se agrupan las enfermedades crónicas degenerativas, éstas crecieron paulatinamente producto de la menor mortalidad infantil y del aumento de la esperanza de vida al nacer. Así afecciones como el cáncer, pasaron de 4,3 a $11,2 \%$ y las cardiovasculares 8,8 a 30,4\%o entre 1935 y 1947. Según Celton: «A medida que la mortalidad descendía, con mayor intensidad desde 1935

31 Carbonetti, A. y Peranovich, A. (2006), La mortalidad infantil en la ciudad de Córdoba entre principios y mediados del siglo XX. En XX Jornadas de Historia Económica, Mar del Plata.

32 Celton, D. E. (1992), La mortalidad en la ciudad de Córdoba (Argentina) entre 1869 y 1990. Boletín de la Asociación de Demografía Histórica, 10 (1), pp. 34-35. 
[...] surgió un 'patrón moderno', similar a los países industrializados». ${ }^{33} \mathrm{En}$ definitiva, existieron cambios en las condiciones de vida de la población que dieron lugar a modificaciones en las problemáticas sanitarias a ser consideradas por los entes públicos.

Vinculado a esto último, en los años treinta se puso en evidencia un aumento sostenido en la demanda de atención sanitaria. En el caso de cuatro de los hospitales de beneficencia existentes en la ciudad de Córdoba, dos generales y dos para la atención de los enfermos de tuberculosis, el ingreso de enfermos aumentó en un 50\% entre 1930 y $1943 .{ }^{34}$ Este fenómeno puede atribuirse a diversas causas. Por un lado, se dieron factores coyunturales como las consecuencias sociales de la crisis económica de comienzo de los años treinta, con sus efectos en el aumento del desempleo y la caída de los ingresos que dificultaron el acceso por medio del mercado a la medicina privada; por otro lado, cambios estructurales como el crecimiento poblacional, el proceso de medicalización, la consolidación de una cultura higiénica ${ }^{35}$ y los cambios en las tendencias de los índices de mortalidad, incidieron en el aumento de las solicitudes de atención en los servicios gratuitos de salud. Como consecuencia de esta demanda ampliada, los hospitales de beneficencia tendieron a gestionar fondos para aumentar sus servicios buscando adecuarse a las necesidades existentes. Un proceso similar se produjo en el ámbito público, donde la expansión de la atención sanitaria respondió a una demanda en constante crecimiento que colapsaba los servicios existentes. Ligado a esto, otro de los fenómenos emparentados a los procesos de cambio en la demanda fue el de la ampliación de la categoría del sujeto de atención de la política sanitaria. En ese sentido, el tradicional destinatario de la beneficencia pública, caracterizado por la indigencia y definido por su invalidez, fue paulatinamente modificado para incluir a todos aquellos cuyas condiciones materiales dificultaban su acceso a la medicina por medio del mercado, en especial, a los trabajadores. ${ }^{36}$

Ahora bien, hay otra serie de factores que permiten explicar también los cambios en la orientación de las políticas sanitarias y están relacionados con

33 Ibid., p. 50.

34 Anuario Estadístico de la Provincia de Córdoba, años 1930-1943.

35 Armus, D. y Belmartino, S. (2001), Enfermedades, médicos y cultura higiénica. En Cattaruzza, A. (dir.), Nueva Historia Argentina. Crisis económica, avance del Estado e incertidumbre política (1930- 1943), t. VII, Buenos Aires, Sudamericana, 283-329.

36 Ortiz Bergia, M. J. (2009), De caridades y Derechos. La construcción de políticas sociales en el interior argentino. Córdoba (1930-1943), Córdoba, Centro de Estudios Históri$\cos , 120$. 
las modificaciones producidas en el mismo seno de la práctica médica, a partir de cambios en las rutinas, en las formas de diagnóstico y de las terapias. Al respecto, es necesario destacar las implicancias que tuvo el desarrollo de los avances científicos en la prevención y atención de muchas enfermedades, como la aplicación de nuevos tipos de análisis, la lenta implementación de los mecanismos de asepsia y antisepsia y el desarrollo de una compleja aparatología de diagnóstico y terapia como los rayos $\mathrm{X}$ que en esos años se consolidaron como dispositivos terapéuticos y de análisis. Esto tuvo profundas consecuencias en las mayores expectativas sociales en torno a la eficacia de la medicina y en la consolidación de una estructura de prestación médica centrada en el hospital. A lo largo de la primera mitad del siglo XX, éste dejó de ser un refugio donde los pobres iban a morir y se convirtió en un espacio preferente de prestación dado el acceso a equipamientos de complejidad y por su capacidad para aglutinar a las nuevas especialidades médicas que se iban estructurando. ${ }^{37}$ Estas cuestiones incidieron en la gestación de modificaciones en la cobertura de los servicios desplegados por el Estado, en el formato y en la orientación que adquirieron las políticas sanitarias públicas provinciales.

Otro elemento explicativo de la mayor envergadura que adoptó el $\mathrm{CPH}$ dentro del campo sanitario y dentro del aparato estatal fue el accionar desplegado por los funcionarios a cargo del mismo a lo largo de esos años. En ese sentido, el común denominador de los dirigentes elegidos para desempeñar su presidencia fue el de personalidades con una destacada trayectoria en el campo médico, permitiendo revestir al organismo de un mayor protagonismo público y a sus nuevas propuestas de intervención de una creciente legitimidad basada en sus saberes científicos. Así, tanto Alejandro Centeno (19301931), Francisco de la Torre (1932-1935) como Guillermo Stuckert (19361943) constituyeron referentes profesionales en el ámbito cordobés, con una importante inserción universitaria a lo largo de las primeras décadas del siglo en el marco de la Facultad de Ciencias Médicas de la Universidad Nacional de Córdoba.

En lo que hace al primero de ellos, Alejandro Centeno se graduó de médico en 1889, se especializó en Francia y luego volvió a Córdoba pasando a formar parte de la planta docente de la Facultad en diversas cátedras como Patología Interna, Enfermedades Nerviosas, primer profesor titular de Enfermedades Infecciosas, en 1904, y, finalmente, se retiró de la docencia como

37 Esto último se puso en evidencia en la creciente organización de sociedades profesionales cordobesas como las sociedades de tisiología, medicina interna, pediatría, oftalmología, dermatología, biología, radiología, cirugía y otorrinolaringología. 
titular de la Cátedra de Clínica Médica. Decano de la Facultad de Medicina entre 1914 y 1918, propició la creación de una Escuela de Odontología, del Instituto Antirrábico y la implantación de las cátedras libres. En el campo médico recibió importantes reconocimientos como miembro de la Academia Nacional de Medicina -1932- y del comité académico de la revista médica del Círculo Médico de Córdoba, institución a la cual pertenecía. ${ }^{38}$ Además, como representante de una de las familias más tradicionales de Córdoba formó parte de la vida pública y social de la provincia en forma constante a lo largo del período de entre siglos. Fue legislador provincial en 1895, 1898-1907, 19141918 y 1926-1930. En su foja de servicios también constó la dirección de hospitales y reparticiones sanitarias públicas como la Asistencia Pública de la ciudad de Córdoba. En ese sentido, constituyó un profesional con una larga trayectoria pública, contando con un importante capital académico, social y político.

El sucesor de Centeno en el cargo de presidente del CPH durante la administración demócrata (1932-1935) fue Francisco de la Torre, un dirigente con menos antecedentes que Centeno pero igualmente bien posicionado en el ámbito académico y profesional de la provincia. El mismo se había recibido en 1906 en la ciudad de Buenos Aires. Durante los años 1910 y 1913 viajó por Europa y Estados Unidos. Entre 1914-1918 fue jefe de servicios de niños del Hospital Rawson en Buenos Aires y llegó a Córdoba en 1918 para desempeñarse como titular de Materia Médica y Terapéutica de la Facultad de Medicina de la Universidad local. A semejanza con Centeno ocupó cargos dirigentes en la Universidad, en 1919 fue nombrado consejero y vicedecano de la Facultad de Medicina, pero renunció en 1920, y en 1921 fue nombrado rector de la Universidad dejando el cargo en 1923. Ese mismo año fue representante del gobierno de Córdoba como delegado al Congreso Panamericano del Niño celebrado en Montevideo y, además, dirigió uno de los dispensarios de lactantes que existían en la ciudad capital.

El siguiente presidente del CPH sería un profesional y dirigente político y universitario de trascendencia. Guillermo Stuckert nació en 1889 y se graduó en Córdoba de farmacéutico en 1909 y de doctor en medicina en 1913. Profesor titular de Química Biológica de la Escuela de Medicina (1919-1946), en 1922 se lo nombró titular de la misma materia en la Universidad del Litoral y en Química Inorgánica en la Escuela de Farmacia (1924-1946), además, estuvo a cargo del Instituto de Química Orgánica de la Universidad de Córdoba. Stuckert también fue un docente e investigador destacado, socio del Círculo

38 Revista Médica del Círculo Médico, julio-agosto 1932, p. 286. 
Médico y socio fundador de la Sociedad de Biología de Córdoba (1934), fue el descubridor de la alfa-fagarina, alcaloide de acción específica sobre la fibrilación del corazón. También tuvo una activa vida política al interior de la Universidad siendo elegido miembro del consejo directivo (1927-1931), vicedecano (1929-1930) y decano (1936-1940). Finalmente, fue un activo militante del Partido Radical de Córdoba siendo electo diputado provincial entre 1919-1921 y 1949-1952. 39

En definitiva, los tres presidentes del organismo que hemos reseñado tuvieron un perfil similar, una activa vida académica en la docencia universitaria, una constante práctica médica en establecimientos hospitalarios de la ciudad capital y una intensa vida política, en los tres casos dentro del espacio universitario, ocupando a los largo de más de tres décadas los máximos cargos políticos de esa institución. Finalmente, en lo que hace a Centeno y a Stuckert a través de una vida política comprometida activamente a través no sólo de la función pública como presidentes del $\mathrm{CPH}$, sino también a través de importantes cargos representativos en sus respectivos partidos. Por tanto, los tres presidentes constituyeron nexos privilegiados entre la práctica médica, la formación académica y la labor pública. Podemos señalar entonces que una de las características de estos tres técnicos-políticos fue su alternancia entre espacios de producción de conocimiento social y de políticas sociales. ${ }^{40}$ En los tres casos su significativa trayectoria universitaria permite suponer que su posicionamiento en el ámbito público fue el resultado del prestigio y la legitimidad acumulados a partir de su labor docente, una estrecha relación entre el saber y el poder donde su participación del aparato estatal constituyó una transferencia de sus acreditaciones académicas. Como consecuencia de ello, los saberes adquiridos por estos funcionarios permitieron revestir al $\mathrm{CPH}$ de una mayor jerarquía institucional, potenciando los proyectos presentados, revistiendo de legitimidad científica las demandas de recursos y favoreciendo el acceso a los aparatos públicos de las innovaciones médicas. No obstante, como muestran los casos de Centeno y Stuckert, para acceder a las máximas jerarquías del aparato estatal no eran suficientes sus acreditaciones universitarias sino una larga trayectoria en los círculos partidarios. Entonces, las identificaciones políticas fueron una condición necesaria aunque no suficiente para acceder a la dirección del aparato estatal.

39 AAVV (1955), Quien es quien en la Argentina: Biografías contemporáneas, Buenos Aires, G. Kraft ltda., p. 600.

40 Neiburg, F. y Plotkin, M. (comp.) (2004), Intelectuales y expertos. La constitución del conocimiento social en la Argentina, Buenos Aires, Paidós, pp. 20-25. 
Finalmente, un último elemento que parece necesario revisar es el relativo a lo que podemos denominar como un clima de época favorable al intervencionismo y que dio sustento a la mayor participación estatal en el campo de la salud. En ese sentido, las experiencias de intervencionismo que se desplegaron a nivel mundial como respuestas a la crisis económica de comienzos de los años treinta, generaron un fuerte consenso entre los contemporáneos sobre la necesaria participación estatal en la regulación económica y social. En el contexto cordobés, a partir de 1933 y 1934 incluso sectores más bien liberales articularon discursos donde se reconocía la necesaria intervención del Estado. Es dentro de ese universo conceptual donde la ampliación de la participación estatal en el campo sanitario debe ser entendida, dando legitimidad a la creciente intromisión estatal en esferas consideradas anteriormente como ajenas a sus funciones.

Esas modificaciones se interrelacionaron a su vez con la difusión de discursos sobre la salud/enfermedad que circularon con profusión durante esos años e hicieron de la intervención sanitaria un accionar privilegiado en pos del progreso social. Las investigaciones realizadas han puesto en evidencia cómo a lo largo de las primeras décadas del siglo XX se produjo un desplazamiento conceptual en la definición de los procesos de salud/enfermedad, desde perspectivas definidas en términos liberales y que asumían las enfermedades como resultado de conductas individuales, hacia una mirada social de la salud, pensada como producto de condiciones materiales adversas y factibles de ser neutralizadas mediante el accionar público. En ese contexto el interés por la preservación del «capital humano», entendido por las condiciones óptimas de la población en procura de asegurar el progreso de la sociedad, constituyó la piedra angular alrededor de la cual se articularon las propuestas tendientes a mejorar los servicios asistenciales de salud. En un mensaje uno de los gobernadores del período, el Dr. Amadeo Sabattini, señaló: «el derecho y la moral contemporáneos, al fundamentar por encima de todo el derecho a la vida, ha creado a los Estados el deber consiguiente de asegurar su desarrollo dentro de las mejores condiciones, no sólo en bien del individuo, sino como un imperativo social para la supervivencia del conglomerado humano y para su supervivencia en el futuro. $\rangle^{41}$

Implicadas en estos cambios tendrían marcada incidencia las perspectivas eugénicas que justificaban la intervención estatal con la meta de modificar las condiciones sanitarias de la sociedad. En ese sentido, los debates sobre la cantidad y la calidad de la población tuvieron una marcada trascendencia en

41 Diario de Sesiones de la Cámara de Diputados de la Provincia de Córdoba, 18/8/36, p. 823. 
el ámbito público provincial y nacional. ${ }^{42}$ Incluso, el caso cordobés fue una de las expresiones más importantes del impacto de la eugenesia en las políticas públicas en la Argentina de entreguerras, a través de la implantación simultánea de mecanismos voluntarios y coercitivos de mejoramiento racial. En ese sentido, a diferencia del contexto nacional, en los años treinta el gobierno provincial crearía en procura de contrarrestar los efectos de las enfermedades venéreas, un dispositivo de aislamiento y reclusión compulsiva de las prostitutas en pos de su «curación», el Sanatorio de Previsión Social. ${ }^{43}$ De todos modos, el mayor impacto de las perspectivas eugénicas se puso en evidencia en la fundamentación de políticas sanitarias de carácter inclusivo centradas en la infancia, como el desarrollo de la Puericultura en el ámbito de las Gotas de Leche, con la instrucción de las madres sobre el mejor modo de cuidar a sus pequeños, y en el ámbito educativo a través de la selección y el posterior tratamiento alimenticio, médico y físico de los denominados «niños débiles». Finalmente, podemos aseverar que en términos más amplios la eugenesia funcionó en el contexto local como un discurso legitimador más bien difuso de las innovaciones en política sanitaria, dado que permitió un consenso común entre los distintos conjuntos políticos alrededor de la idea que la salud de un pueblo era su capital más importante.

Ahora bien, durante esos años el progreso, el crecimiento y el fortalecimiento de la nación fueron núcleos argumentales que sustentaron las innovaciones institucionales en el campo de la salud pública, sin embargo, esos tópicos también establecieron límites a la mayor participación estatal en el campo sanitario. Eso se expresó en las decisiones políticas respecto a la distribución de los recursos públicos y en el escaso protagonismo presupuestario del área de salud.

Como hemos señalado anteriormente, la salud en el esquema público provincial adquirió una escasa relevancia presupuestaria. La salud era relegada y los funcionarios a cargo del área se mostraron contrariados con esta política. Pero para explicar esto es necesario situarse en el contexto en el que vivían

42 Para seguir la discusión historiográfica actual sobre la incidencia de la eugenesia en las políticas públicas en la Argentina, ver: MIRANDA M. A. (2003), La antorcha de cupido: eugenesia, biotipología y eugamia en Argentina, 1930-1970, Asclepio, vol. LV, 2, 231-255; DI Liscia M. S. (2008), Reflexiones sobre la «Nueva Historia Social» de la salud y la enfermedad en Argentina, CARBOnETTI A. y GONZÁLEZ-LEANDRI R. (ed.), Historias de salud y enfermedad en América latina siglos XIX y XX, CEA-CONICET, Córdoba, 15- 47.

43 Rimonda, N. D. (2008), Tecnologías de control social en defensa de la salud pública. La prostitución en la ciudad de Córdoba entre 1936-1954, Trabajo Final de Licenciatura en Historia, Universidad Nacional de Córdoba, Inédito. 
los contemporáneos en el interior del país, en donde si la salud de la población constituía un pilar para el progreso social y económico, no por ello era el único, el más eficaz o el políticamente más redituable. En cambio, para la dirigencia política la seguridad, la educación y las obras públicas fueron políticas más estrechamente vinculadas con la generación del progreso económico y social del territorio, a través de la preservación de la propiedad privada urbana y rural, la puesta en valor de nuevas tierras para la agricultura a través del riego, la formación de los futuros ciudadanos y el mejoramiento de las vías de comunicación y de transporte. La salud constituía un elemento más de las políticas públicas que aseguraban el crecimiento económico y el progreso político, pero no el más importante. Eso explica el porqué si bien existieron cambios y rupturas respecto al lugar que ocuparon las políticas sanitarias, existieron a su vez permanencias, como la falta de recursos humanos y materiales a disposición del $\mathrm{CPH}$, escasez de infraestructura sanitaria en vastos espacios y en algunos casos imprevisión en la atención de algunas afecciones infectocontagiosas.

\section{A MODO DE CONCLUSIÓN}

El propósito inicial de este trabajo fue el de poner en evidencia trayectorias diferentes en la estructuración de las políticas sanitarias en la Argentina que permitieran complejizar el proceso de construcción del Estado interventor en el país. Para ello nos abocamos a analizar el aparato sectorial dedicado a la atención de las políticas sanitarias de una provincia del interior del país, Córdoba, identificando los cambios y continuidades que se produjeron en los años treinta en su infraestructura y en la orientación de sus intervenciones sanitarias.

En torno a ese problema pudimos constatar cómo a partir de mediados de los años veinte y más claramente en los treinta, se produjeron modificaciones institucionales en los aparatos públicos provinciales que implicaron su desplazamiento desde una perspectiva liberal y defensiva de la salud hacia una perspectiva social y asistencial/preventiva, que modificó los mecanismos y los alcances de la intervención estatal en las condiciones sanitarias de la población. Estos procesos se expresaron en la extensión de los servicios médicos públicos, el desplazamiento de la preocupación por la salubridad urbana, por la atención asistencial de las zonas rurales, la consolidación de políticas de prevención y la construcción paulatina de nuevas capacidades para los aparatos estatales tendientes a permitir una mayor autonomía de acción y una 
más amplia penetración del territorio. Para comienzos de los años cuarenta estos procesos modificarían la participación de las estructuras estatales provinciales en el campo de la salud, desplazándolas desde una actitud liberal, característica de las primeras décadas del siglo XX, hacia un mayor protagonismo que implicaría la ampliación de sus intervenciones y la modificación de sus objetivos y orientaciones.

En la base de estos procesos se entrecruzaron una serie compleja de causas. Primero, cambios en las condiciones materiales de la población y en sus demandas de salud, que impactaron en el interés político por ampliar la infraestructura gratuita al alcance de los sectores de menores ingresos, en especial, en las zonas con escasa disponibilidad de acceso a la prestación de atención médica. Un segundo elemento causal fueron las innovaciones en las prácticas médicas que renovaron la prestación de los servicios sanitarios, tales como el creciente proceso especialización y la aplicación de novedades científicas y tecnológicas en el diagnóstico y las terapias. Asimismo, el mayor protagonismo estatal en el campo de la salud fue en parte el resultado de los dirigentes que se integraron a la burocracia estatal en esos años. En ese sentido, los funcionarios sanitarios a cargo del aparato estatal se destacaron por su extensa trayectoria docente y política en el ámbito universitario, experiencia que cimentó su prestigio personal y el de sus saberes adquiridos, generando una mayor jerarquía de las reparticiones de salud dentro del organigrama público y su mayor capacidad para potenciar a la salud en la agenda política. Finalmente, un último fenómeno vinculado a los anteriores es el de la consolidación de modificaciones en los marcos conceptuales de los sectores dirigentes a través de los cuales se pensaba el problema de la salud, las políticas sociales y el Estado. La conformación de un consenso favorable a la intervención estatal en la salud fue el resultado de diferentes discursos que circulaban en esos años sobre el valor del capital humano/trabajador, las teorías eugénicas extensamente aceptadas en las discusiones políticas del momento y las perspectivas reformistas de la «cuestión social» arraigadas en el ámbito local entre liberales, progresistas y conservadores.

Recibido: 15 de noviembre de 2010

Aceptado: 20 de octubre de 2011 